\title{
A new factor in the circadian control of barley chloroplast development
}

\author{
PingPing Fang ${ }^{1}$, Nurhayati Razak ${ }^{2}$, Barbara Karpinska ${ }^{1}$, Tracy Lawson ${ }^{3}$, JQ yu ${ }^{4}$, and \\ Christine Foyer ${ }^{1}$ \\ ${ }^{1}$ University of Birmingham \\ ${ }^{2}$ University of Leeds \\ ${ }^{3}$ University of Essex Department of Biological Sciences \\ ${ }^{4}$ Affiliation not available
}

July 13, 2020

\begin{abstract}
Photoperiod and circadian controls play crucial roles in the regulation of chloroplast biogenesis. To understand more about the regulation of this process, we compared the greening of the first leaves of wild type barley and two WHIRLY1 (WHY1)-deficient lines. Seedlings were grown in darkness for 4 days prior and then exposed to light at the beginning of the photoperiod on the 5th day or under standard photoperiod conditions. The accumulation of chlorophyll, as well plastid-encoded photosynthetic transcripts and proteins was delayed in the WHY1-deficient lines under standard photoperiod conditions because of defects in plastid gene expression, ribosomal processing and photosynthetic protein accumulation. The acquisition of full photosynthetic capacity was delayed by about 11 days in the first leaves and the newly forming leaves of the WHY1-deficient lines compared to the wild type. However, the light-dependent accumulation of pigments, transcripts and photosynthetic proteins was similar in all lines when etiolated seedlings were exposed to light. These results demonstrate that WHY1 is required for the integration of photoperiod-dependent signalling and chloroplast development in barley leaves.
\end{abstract}

\section{INTRODUCTION}

Light not only drives photosynthesis but it is also one of the major environmental signals that regulates plant growth and development. In darkness the germinating seeds undergo a skotomorphgenesis program that results in seedlings with long hypocotyls and closed cotyledons that contain non-green plastids (etioplasts), which develop from proplastids in the meristem. Upon the perception of light signals, a de-etiolation process occurs that is marked by the synthesis of chlorophyll and the transition from etioplasts to chloroplasts. However, when seedlings are germinated in the light, proplastids develop directly into chloroplasts without the intermediate etioplast stage. Phytochrome and cryptochrome photoreceptors are responsible for light perception and the transduction of light signals that regulate development in photomorphogenesis (Neff et al., 2000 Schäfer and Bowler 2002).

Phytochrome A is the primary photoreceptor mediating far-red light signalling through FAR-RED ELONGATED HYPOCOTYL3 (FHY3) and FAR-REDIMPAIRED RESPONSE1 (FAR1), which function as positive regulators of early greening (Wang et al., 2002). FAR1 is a transposase-related transcription factor that activates the expression of FAR-RED ELONGATED HYPOCOTYL1 and the FHY1-LIKE PROTEIN . These proteins promote the nuclear translocation of phytochrome A resulting in the activation of phytochrome A-mediated gene expression, which includes genes related to chloroplast division and chlorophyll synthesis (Tang et al., 2012). The FAR1 protein also acts as a positive regulator of ABA signalling in Arabidopsis , enabling adaptation to environmental stresses (Tang et al., 2013). During chloroplast biogenesis 
light promotes chlorophyll synthesis to support light harvesting in photosynthesis. The major rate-limiting step in chlorophyll biosynthesis is the formation of 5-aminolevulinic acid (ALA). FHY3 and FAR1 regulate the early greening process during de-etiolation by binding to promoter of HEMB1, which encodes 5 -aminolevulinic acid dehydrogenase (ALAD), an enzyme that converts ALA into protoporthyrin IX early in the chlorophyll biosynthetic pathway. Upon perception of light phytochrome A, FHY3 /FAR1 are expressed, increasing HEMB1 transcript levels and ALAD protein levels. This promotes protochlorophyllide (Pchlide) synthesis and chlorophyll accumulation. In darkness, Pchlide is accumulated in the etioplasts because of the light requirement for activation of the enzyme NADPH:protochlorophyllide oxidoreductase (POR), which converts Pchlide to chlorophyllide (Solymosi and Schoefs, 2010). FAR1 also resets the circadian clock in response to red light, a process that is downstream of multiple phytochrome photoreceptors. FAR1 binds to a FHY3/FAR1 binding site (FBS) that activates the expression of elongation factor 4 (ELF4) and circadian clock associated 1 (CLOCK ASSOCIATED1 ; CCA1) to reset the clock (Liu et al., 2020).

Chloroplast development also requires extensive chloroplast tonucleus retrograde signalling, which enables the coordination of the nuclear and chloroplast transcriptional activities that are required for the assembly of the photosynthetic apparatus (Börner, 2017; Chan et al., 2016). Retrograde signalling ensures the balanced and co-ordinated expression of nuclear-encoded PHOTOSYNTHESIS ASSOCIATED NUCLEAR GENES (PhANGs), a process that relies on information supplied by positive signals provided by the developing plastids (Dubreuil et al., 2018). Defects in plastid development are signalled through retrograde signalling to attenuate the expression of photosynthetic genes in the nuclei. Retrograde signalling pathways such as the plastid-localized GENOMES UNCOUPLED (GUN) retrograde signalling pathway (Susek et al 1993) that regulates the expression of nuclear-encoded PhANGs, have been extensively studied. ThePhANGs, suite of genes encode components of the photosynthetic electron transfer chain such as the LIGHT HARVESTING CHLOROPHYLL A/B BINDING PROTEIN COMPLEX (LHC) or PLASTOCYANIN (Susek et al 1993, Hess et al., 1994; Ruckle et al., 2007). GUN1 is multifunctional protein that regulates the synthesis of tetrapyroles, which are mediators of one of the biogenic chloroplast-to-nucleus retrograde signalling pathways (Shimizu et al., 2019). Loss of GUN1 protein uncouples PHANGexpression from the developmental state of the plastids due to the impaired protein import into the plastids (Wu et al., 2019). However, the biogenic pathway of retrograde signalling is still poorly understood (Kleine et al., 2009)

The dually-located protein WHIRLY1 (WHY1) has been proposed to function as a retrograde protein signal (Comadira et al., 2015). The differential localisation of WHY1 between the chloroplasts and nuclei is mediated by protein phosphorylation, a process catalysed by the calcineurin B-like-Interacting Protein Kinase14 (CIPK14; Ren et al., 2017). Interestingly, CIPK14 was shown to be involved in phytochrome A-mediated far-red light inhibition of greening in Arabidopsis (Qin et al., 2010). We have previously reported that the fully developed WHY1 deficient barley leaves accumulate more chlorophyll and have higher chlorophyll a/b ratios than controls (Comadira et al., 2015). Crucially, lack of WHY1 functions led to enhanced levels of FAR1 transcripts, as well as high levels of transcripts encoding chloroplast ribosomes, the thylakoid NADH dehydrogenase and cytochrome b/f complexes (Comadira et al., 2015).

To investigate the functions of WHY1 in chloroplast development further, we compared the greening of two WHY1-deficent barley seedlings under standard day/night photoperiod conditions and de-etiolation. The leaves of the seedlings grown in standard light conditions showed delayed in greening (by about 9 days) and they accumulated less plastid-encoded photosynthetic proteins. However, during de-etiolation the WHY1-deficient seedlings were able to synthesize pigments and photosynthetic proteins in a similar manner to the wild type. In addition, WHY1 deficient leaves showed enhanced accumulation of the LHCB protein both during de-etiolation and under standard photoperiod growth conditions. These results taken together with our earlier findings (Comadira et al., 2016) suggest that WHY1 deficient barley seedlings are low light-adapted. Since the exposure of etiolated seedlings to light abolishes phytochrome-mediated circadian controls, we suggest that WHY1 regulates FAR1 in the circadian-dependent control of photosynthesis during plastid development in order to protect photosynthesis in a fluctuating light environment.

\section{MATERIALS AND METHODS}




\section{Plant Material}

Seeds of two independent transgenic barley (Hordeum vulgare L. cv. Golden Promise) lines (W1-1 and W17) with RNAi knockdown of the WHIRLY1 gene and wild type controls were produced in the laboratory (Comadira et al., 2015).

Standard Photoperiod growth conditions.

Barley seeds (1 per pot) were sown in pots in compost (SHL professional potting compost) in controlled environment chambers with a $16 \mathrm{~h}$ light $/ 8 \mathrm{~h}$ dark photoperiod, with an irradiance of $250 \mu \mathrm{mol} \mathrm{m}{ }^{-2} \mathrm{~s}^{-1}, 20^{\circ} \mathrm{C} / 16^{\circ} \mathrm{C}$ day/night temperature regime and $60 \%$ relative humidity. The first leaves on the seedlings were harvested after 7 and 14 days.

\section{Etiolation.}

Barley seeds (1 per pot) were sown in pots in compost (SHL professional potting compost) in a controlled environment chamber. They were grown in continuous darkness for 4 days and then subjected to irradiance $\left(250 \mu \mathrm{mol} \mathrm{m} \mathrm{m}^{-2} \mathrm{~s}^{-1}\right)$ at the beginning of the $5^{\text {th }}$ day, when the seedlings were returned to the above standard photoperiod growth conditions, with a $16 \mathrm{~h}$ light $/ 8 \mathrm{~h}$ dark photoperiod, $20^{\circ} \mathrm{C} / 16^{\circ} \mathrm{C}$ day $/$ night temperature regime and $60 \%$ relative humidity. The first leaves on the seedlings were harvested at the times indicated on the figure legends in the hours following return to standard photoperiod growth conditions.

\section{Leaf Pigments}

Total chlorophyll was extracted from the barley leaves in $80 \%$ (v/v) acetone. Absorbance of chlorophylls was measured at 663 and $646 \mathrm{~nm}$ as described by Lichtenthaler (1987).

\section{$q P C R$}

Reverse transcription of $1 \mu \mathrm{g}$ of RNA into cDNA was performed using the QuantiTect Reverse Transcription Kit (Qiagen). The qPCR was performed using QuantiFast SYBR Green PCR kit (Qiagen) in the presence of $0.5 \mu \mathrm{M}$ primers in a CFX96 thermocycler (Biorad, Hercules, CA, USA) following the manufacturer's instructions. PCR conditions were as follows: incubation at $95{ }^{\circ} \mathrm{C}$ for $5 \mathrm{~min}, 45$ cycles $10 \mathrm{~s}$ $95^{\circ} \mathrm{C}$ and $30 \mathrm{~s} 60^{\circ} \mathrm{C}$. Additionally melting curve analysis was performed at the end of each run to ensure specificity of the products. The same master mix without cDNA was used as negative control. The twostep cycling protocol were programmed as follows: incubation at $95^{\circ} \mathrm{C}$ for $5 \mathrm{~min}$; 40 cycles of amplification comprised of $95^{\circ} \mathrm{C}$ for $10 \mathrm{~s}, 60^{\circ} \mathrm{C}$ for $30 \mathrm{~s}$ and $72^{\circ} \mathrm{C}$ for $30 \mathrm{~s}$. The mean value of three replicates was normalized using actin ( Fwd 5'- CGACAATGGAACCGGAATG-3'; Rev 5'-CCCTT GGCGCATCATCTC3 ')as internal controls. The other primers used were; Whirly 1 (5'-GATGGGAATGGTCGCTTTTT -3'; Rev 5'-CCATGATGTGCGGTATGATG -3'); rbcL (Fwd 5'-TTGGGTTCAAAGCCCTACGTGC-3'; Rev 5'-ACATCCCAATAAAGG ACGGCCA-3') and psbA (Fwd 5'-CAGAAAAGCTTCCTTGACCA-3'; Rev 5'-CAAT GGTGGTCCTTATGAGC-3').

\section{Western Blot analysis}

Total proteins were extracted with protein extraction buffer (Agrisera) supplemented with $5 \mathrm{mM}$ DTT and the cocktail of protease inhibitors to prevent protein degradation. $10 \mu \mathrm{g}$ of proteins were separated on $15 \%$ acrylamide SDS-PAGE, and transferred to $0.45-\mu \mathrm{m}$ nitrocellulose membrane (Amersham 10600003). All proteins apart from WHY1 were detected with rabbit polyclonal primary antibody (Agrisera) and secondary HRP-linked anti-rabbit (1:10000, Agrisera AS09 602). For immunological detection of WHY1, the antibodies were directed toward the synthetic peptide of recombinant HvWhy1 protein (PRQYDWARKQVF) in rabbits and antibodies were affinity-purified (Generon, UK). The specificity of immunodetection was validated using pre-immune sera.

Chlorophyll a fluorescence Imaging Analysis 
Chlorophyll fluorescence imaging was performed using both a customised FluorCam imaging fluorometer, controlled by a computer program called FluorImager (Technologia Ltd., Colchester, UK) and a Walz Chlorophyll Fluorometer IMAGING-PAM M-Series MAXI-Version (System I) based on the Multi Control IMAGCG for large areas up to $10 \times 13 \mathrm{~cm}$. Plants were dark adapted for 30 minutes and placed in the chamber together with pots to image the maximum PSII quantum yield (Fv/Fm).

Statistical Analysis

Data represent the mean \pm standard error of the mean (SE). Statistical analysis was performed by Two-way ANOVA (IBM SPSS Statistics-version 20). The values were considered statistically different when $P$ was $<$ 0.05 .

\section{RESULTS}

The WHY1-deficient barley leaves develop in a similar manner to the wild type except that the greening of each emerging leaf is delayed in the absence of WHY1 (Fig. 1, A, B). However, greening was similar in all lines when the seedlings were first grown in the dark for 4 days and then transferred to the light at the beginning of the photoperiod under standard day/night grown regime (Fig. 1C-H).

The efficiency of photosynthesis was determined by chlorophyll a florescence imaging of the seedlings. The dark-adapted Fv/Fm ratios were much lower in the emerging leaves of W1-7 leaves than the wild type under standard photoperiod conditions (Fig. 2). The efficiency of photosynthesis observed in the first leaves of the W1-7 seedlings attained maximal values only after 9 days, suggesting a delay in greening compared to the wild type in the growth conditions used in these experiments. However, the delay in the establishment of photosynthesis was observed in each emerging lead especially in the base regions of the leaves as observed for example in the 11-day-old W1-7 seedlings (Fig. 2).

All sections of the 7 days old W1-1 and W1-7 leaves had significantly less chlorophyll than the wild type (Fig. 3A). However, the chlorophyll contents of 14 day old W1-1 and W1-7 leaves were much higher and similar to the wild type (Fig. 3B). When seedlings were grown in the dark for 4 days prior to the onset of illumination, the light-dependent accumulation of chlorophyll was similar in all the lines (Fig. 3C).

The chlorophyll a/b ratios were similar in the developing leaves of all lines at 7 days (Fig. 4A) and 14 days (Fig. 4B) after germination. However, when seedlings that had been grown in the dark for 4 days were transferred to light the chlorophyll a/b ratios of the W1-1 and W1-7 leaves were significantly higher than the wild type after 8 hours exposure to light (Fig. 4C). At later stages of de-etiolation (16h, 32h, 40h and $56 \mathrm{~h}$ ) however, the chlorophyll a/b ratios were similar in all lines (Fig. 4C)

Since monocotyledonous leaves show a developmental gradient from base to tip, the first leaves of 7 and 14 day old seedlings were divided into three sections: base, middle and tip, for further analysis. The levels of WHY1 protein were highest in the basal sections of developing leaves and lowest in the tip sections at 7 days after germination (Fig. 5A). In contrast, the levels of the WHY1 protein were low in all the sections of 14 day old leaves (Fig. 5B). The levels of LHCB1, LHCB2 and RBCS proteins were similar or slightly higher in all regions of the leaves of 7 (Fig. 5A) and 14 (Fig. 5B) day old W1-1 and W1-7 seedlings than the wild type under standard photoperiod conditions. In contrast, the D1 protein, the RPS1 and RBCL proteins were much less abundant in the leaves of 7 day old W1-1 and W1-7 seedlings than the wild type under these conditions (Fig. 5A). The levels of most of these proteins were similar in all lines at 14 days (Fig. 5B). However, the LHCB2 protein was more abundant in the base sections of the 14 day old W1-1 and W1-7 leaves than the wild type, as well as in the middle and tip sections of the W1-7 leaves relative to the wild type (Fig. 5B).

In contrast to the marked delay in chloroplast protein accumulation observed in plants grown under standard photoperiod conditions, the levels of chloroplast proteins increased in a similar timescale in the hours after 4 day old etiolated seedlings were exposed to light (Fig. 6). The levels of the nuclear encoded PSAC subunit of photosystem I (Fig. 6A) and the light harvesting proteins LHCA1 (Fig. 6C) and LHCB1 (Fig. 6D) showed a similar manner of accumulation in all the lines. However, the time-dependent accumulation of the 
chloroplastic ribosomal protein called RPL20 (Fig. 6B) and the D1protein (Fig. 6E) was delayed by several hours in the W1-1 and W1-7 leaves relative to the wildtype.

The levels of transcripts encoding WHY1 increased over the first 8 hours of illumination in the first leaves of the etiolated seedlings and then decreased once more (Fig. 7A). The levels of $P S B A$ (Fig. 7B) and $R B C L$ transcripts (Fig. 7C) showed similar patterns of light-dependent accumulation in all lines.

\section{DISCUSSION}

A number of functions for the DNA-binding WHY1 protein have been described in plants. WHY1 functions as a transcription factor in the nucleus, where it regulates the expression of pathogen response and senescence genes (Desveaux et al., 2004; Xiong et al., 2009; Miao et al., 2013; Krupinska et al., 2019). In chloroplasts, WHY1 maintains the stability of organelle genome (Marechal et al., 2008) and interacts with telomere repeat sequences, such as $p s b A$ and the TAC complex. WHY1 also interacts with WHY3 in the chloroplasts of dicotyledonous species to maintain organelle genome stability (Maréchal et al., 2008; Guan et al., 2018). It also has chaperone functions in RNA processing in chloroplasts (Pfalz et al., 2006; Prikryl et al., 2008; Meloneck et al., 2010; Świda-Barteczka et al., 2018). WHY1 is also associated with the thylakoid membrane and has putative functions in photosynthesis (Isemer et al., 2012; Foyer et al., 2014; Comadira et al., 2015; Huang et al., 2017; Guan et al., 2018; Lin et al., 2019). An earlier study on knockdown maize ZmWhy1 mutants, which have a pale green phenotype (Prikryl et al., 2008) suggested that WHY1 is involved in chloroplast biogenesis. The data presented here shows that WHY1 protein levels were highest in the basal regions of 7 day old wild type leaves grown under standard photoperiod conditions and lowest in the tip, suggesting that the WHY1 protein is most abundant during chloroplast development. Moreover, WHY1 transcripts increased only in the first hours of leaf greening following etiolation and then decreased once more.

The WHY1-deficient leaves showed delayed greening relative to the wild type when seedlings were grown under standard photoperiod conditions. In contrast, when the seedlings were first grown in continuous darkness and then subjected to light there was little or no delay in the accumulation of chlorophyll and photosynthetic proteins in the first leaves of the WHY1-deficient seedlings. We have previously shown that the fully developed W1-1 and W1-7 leaves have similar rates of photosynthetic carbon assimilation to the wild type (Comadira et al., 2015). The mature WHY1-deficient leaves accumulate more chlorophyll and have higher chlorophyll a/b ratios than the wild type with enhanced levels of transcripts encoding photosynthetic proteins including the thylakoid NADH dehydrogenase and cytochrome $\mathrm{b} / \mathrm{f}$ complexes. These changes in mature leaves may be linked to a failure to regulate cpDNA copy number, which is greatly increased in WHY1-deficient leaves (Karpinska et al., 2017). The data presented here show that higher levels of the LHCB2 protein were present in the base sections of the 14 day old W1-1 and W1-7 leaves than the wild type, as well as in the middle and tip sections of the W1-7 leaves relative to the wild type. This finding agrees with a recent report (Krupinska et al., 2019) that WHY1-deficient lines over-accumulate LHC proteins even at the early stages of chloroplast development.

The data presented here shows that proteins encoded by transcripts arising from the plastid genome are less abundant in the developing WHY1-deficient barley leaves. In contrast, chloroplast proteins encoded by nuclear genes are more abundant in the WHY1-deficient leaves. The lack of functional chloroplast ribosomes as evidenced here by the delayed accumulation of the large ribosomal subunit (50S) is thought plays a major contribution to the delay in chloroplast development (Prikryl et al., 2008). As a result of the lack of synchrony between plastid and nuclear gene expression, the establishment of photosynthesis is delayed by about 11 days in W1-1 and W1-7 leaves compared to the wild type under standard photoperiod conditions. Chloroplast genes are transcribed as operons, the generation of various mono- and poly-cistronic RNA molecules are regulated by complex post-transcriptional RNA processing mechanisms (Morley et al., 2016; Sun et al., 2016). Since the WHY1 protein does not specifically bind to the operon containing these RNAs, it is likely that WHY1 functions directly through interactions with other RNA-binding proteins that function in the synthesis, assembly or stability of $50 \mathrm{~S}$ ribosomal subunits in chloroplasts. 
Taken together, the above observations show that WHY1 fulfils functions in the accumulation of plastome transcripts and proteins in developing barley chloroplasts but that this requirement largely disappears when the developing leaves are first exposed to a long dark period before experiencing light. These findings suggest that the WHY1 protein fulfils a role in the photoperiod-dependent or circadian regulation of chloroplast development because delayed greening is only observed when the WHY1-deficient seedlings are grown under standard photoperiod conditions. Transcriptome profiling analysis has shown that the expression of relatively few genes is changed in leaves as a result of the loss of WHY1 functions. However, the levels of FAR1 transcripts are always significantly higher in the WHY1-deficient leaves than the wild type (Comadira et al., 2015). FAR1 binds to the promoter of HEMB1, which encodes 5-aminolevulinic acid dehydratase in the chlorophyll biosynthetic pathway, activating expression (Tang et al., 2012). Moreover, FAR1 is also important in the resetting of the circadian clock by red light downstream of multiple phytochrome photoreceptors (Liu et al., 2020). It is tempting to suggest that WHY1 functions in the circadian regulation of chloroplast development through the repression FAR1 expression.

\section{ACKNOWLEDGEMENTS}

We thank BBSRC (UK) for financial support (BB/N004914/1). N.R. thanks the Ministry of Rural and Regional Development Malaysia for a PhD scholarship at the University of Leeds, UK; P.P.F. thanks the International Cooperative PhD Research Program funded by Zhejiang University and Dr.Jingquan Yu.

\section{REFERENCES}

Börner T (2017) The discovery of plastid-to-nucleus signaling-a personal perspective.Protoplasma 254 : 18451855 .

Chan KX, Phua SY, Crisp P, McQuinn R, Pogson BJ (2106) Learning the languages of the chloroplast: retrograde signaling and beyond.Annual Review of Plant Biology 67 : 25-53.

Comadira G, Rasool B, Karpinska B, Márquez García B, Morris J, Verrall S R, Bayer M, Hedley PE, Hancock RD, Foyer CH (2015) WHIRLY1 functions in the control of responses to N-deficiency but not aphid infestation in barley (Hordeum vulgare). Plant Physiology 168 : 1140-1151.

Desveaux D, Subramaniam R, Després C, Mess JN, Lévesque C, Fobert PR, Dangl JL, Brisson, N (2004) A "Whirly" transcription factor is required for salicylic acid dependent disease resistance in Arabidopsis.Developmental Cell 6 : 229-240.

Dubreuil C, Jin X, de Dios Barajas-López J, Hewitt TC, Tanz SK, Dobrenel T, Schröder WP, Hanson J, Pesquet E, Grönlund A, Small I, Strand A (2018) Establishment of photosynthesis through chloroplast development is controlled by two distinct regulatory phases. Plant Physiology 176 : 1199-1214.

Foyer CH, Karpinska B, Krupinska K (2014) The functions of WHIRLY1 and REDOX-RESPONSIVE TRANSCRIPTION FACTOR 1 in cross tolerance responses in plants: A hypothesis. Philosophical Transactions of the Royal Society B: Biological Sciences 369 : 20130226.

Guo H, Feng P, ChiW, Sun X, Xu X, Li Y, Ren D, Lu C, David Rochaix J, Leister D, Zhang L (2016) Plastid-nucleus communication involves calcium-modulated MAPK signalling. Nature Communications7 : 12173.

Guan Z, Wang W, Yu X, Lin W and Miao Y (2018) Comparative Proteomic Analysis 723 of Coregulation of CIPK14 and WHIRLY1/3 Mediated Pale Yellowing of Leaves in 724 Arabidopsis. International Journal of Molecular Sciene 19 :2231

Hess W R, Muller A, Nagy F, Borner T ( 1994) Ribosome-deficient plastids affect transcription of lightinduced nuclear genes: genetic evidence for a plastid-derived signal. Molecular and GeneralGenetics 242 : 305-312.

Huang D, Lin W, Deng B, Ren Y, Miao Y. 2017. Dual-Located WHIRLY1 Interacting 730 with LHCA1 
Alters Photochemical Activities of Photosystem I and Is Involved in 731 Light Adaptation in Arabidopsis.International Journal of Molecular Science 18: 2352.

Isemer R, Krause K, Grabe N, Kitahata N, Asami T, Krupinska K( 2012) Plastid located WHIRLY1 enhances the responsiveness of Arabidopsis seedlings toward abscisic acid. Fronteirs in Plant Science $\mathbf{3}$ : Article 283.

Karpinska B, Owdah Alomrani S, Foyer CH (2017) Inhibitor-induced oxidation of the nucleus and cytosol in Arabidopsis thaliana: Implications for organelle to nucleus retrograde signalling.Philosophical Transactions of the Royal Society B: Biological Sciences 372 : 20160392.

Kleine T, Voigt C, Leister D (2009) Plastid signalling to the nucleus: messengers still lost in the mists? Trends in Genetics 25, 185-192.

Krupinska K., Oetke S, Desel C, Mulisch M, Schäfer A, Hollmann J, Kumlehn J, Hensel G ( 2014) WHIRLY1 is a major organizer of chloroplast nucleoids. Fronteirs in Plant Science $\mathbf{5}$ : article 432.

Krupinska K, Braun S, Nia MS, Schäfer A, Hensel G, Bilger W (2019) The nucleoid-associated protein WHIRLY1 is required for the coordinate assembly of plastid and nucleus-encoded proteins during chloroplast development. Planta 249 : 1337-1347.

Lichtenthaler HK. (1987) Chlorophylls and carotenoids - pigments of photosynthetic biomembranes. Methods in Enzymology 148 : 350-382.

Lin W, Huang D, Shi X, Deng B, Ren Y, Lin W and Miao Y (2019) H2O2 as a Feedback Signal on DualLocated WHIRLY1 Associates with Leaf Senescence in Arabidopsis. Cells 8 :1585.

Liu Y, Ma M, Li G, Yuan L, Xie Y, Wei H, Ma X, Li Q, Devlin PF, Xu X, Wang H (2020) Transcription Factors FHY3 and FAR1 Regulate Light-Induced CIRCADIAN CLOCK ASSOCIATED1 Gene Expression in Arabidopsis. Plant Cell DOI: https://doi.org/10.1105/tpc.19.00981).

Melonek J, Mulisch M, Schmitz-Linneweber C, Grabowski E, Hensel G, Krupinska K (2010) Whirly1 in chloroplasts associates with intron containing RNAs and rarely co-localizes with nucleoids. Planta232 : 471-481.

Marechal A, Parent J-S, Sabar M, Veronneau-Lafortune F, Abou-Rached C, Brisson N (2008) Overexpression of mtDNA-associated AtWhy2 compromises mitochondrial function. BMC Plant Biology $8: 42$.

Miao Y, Jiang JJ, Ren YJ, Zhao ZW (2013) The Single-stranded DNA-binding protein WHIRLY1 represses WRKY53 expression and delays leaf senescence in a developmental stage-dependent manner in Arabidopsis. Plant Physiology 163: 746-756.

Morley SA, Nielsen BL (2016) Chloroplast DNA copy number changes during plant development in organelle DNA polymerase mutants. Front Plant Science. 7, Article 57.

Neff, M.M., Fankhauser, C., and Chory, J (2000) Light: An indicator of time and place. Genes and Devopment 14: $257-271$

Pfalz J, Liere K, Kandlbinder A, Dietz KJ, Oelmuller R (2006) pTAC2, -6, and -12 are components of the transcriptionally active plastid chromosome that are required for plastid gene expression. Plant Cell 18 :176-197.

Prikryl J, Watkins KP, Friso G, van Wijk KJ, Barkan A ( 2008) A member of the Whirly family is a multifunctional RNA- and DNA-binding protein that is essential for chloroplast biogenesis. Nucleic Acids Resaerch 36 : 5152-5165.

Qin Y, Guo M, Li X, Xiong X, He C, Nie X, Liu X (2010) Stress responsive gene CIPK14 is involved in phytochrome A-mediated far-red light inhibition of greening in Arabidopsis. Science China Life Sciences $\mathbf{5 3}$ :1307-1314. 
Ren Y, Li Y, Jiang Y, Wu B, Miao Y (2017) Phosphorylation of WHIRLY1 by CIPK14 806 shifts its localization and dual functions in Arabidopsis. Molecular Plant 10 : 749-807 763.

Ruckle M E, DeMarco S M, Larkin R M ( 2007) Plastid signals remodel light signaling networks and are essential for efficient chloroplast biogenesis in Arabidopsis. Plant Cell 19 : 3944-3960.

Schafer E and Bowler C (2002) Phytochrome-mediated photoperception and signal transduction in higher plants.EMBO Reports 3 : 1042-1048.

Shimizu T, Kacprzak SM, Mochizuki N, Nagatani A, Watanabe S, Shimada T, Tanaka K, ${ }^{e}$ Hayashi Y, Arai M, Leister D, Okamoto H, Terry M J, Masuda T (2019) The retrograde signaling protein GUN1 regulates tetrapyrrole biosynthesis.Proceedings of the National Academy of Sciences U S A. 116 : 24900-24906.

Solymosi K and Schoefs B (2010) Etioplast and etio-chloroplast formation under natural conditions: The dark side of chlorophyll biosynthesis in angiosperms. Photosynthesis Research 105 :143-66.

Susek R E, Ausubel FM, Chory J ( 1993) Signal transduction mutants of Arabidopsis uncouple nuclear CAB and RBCS gene expression from chloroplast development. Cell 74 : 787-799.

Sun A Z, Guo FQ. 2016 Chloroplast retrograde regulation of heat stress responses in plants. Front. Plant Sci ence 7 : Article 398.

Świda-Barteczka A, Krieger-Liszkay A, Bilger W, Voigt U, Hensel G, Szweykowska-Kulinska Z, Krupinska K (2018) The plastid-nucleus located DNA/RNA binding protein WHIRLY1 regulates microRNA-levels during stress in barley (Hordeum vulgare L.). RNA Biology 15 :886-891.

Tang W, Wang W, Chen D, Ji Q, Jing Y, Wang H, Lin R (2012) Transposase-Derived Proteins FHY3/FAR1 Interact with PHYTOCHROME-INTERACTING FACTOR1 to Regulate Chlorophyll Biosynthesis by Modulating HEMB1 during Deetiolation in Arabidopsis. Plant Cell24 : 1984-2000.

Tang W, Ji Q, Huang Y, Jiang Z, Bao M, Wang H, Lin R ( 2013) FHY3 and FAR1 transcription factors integrate light and abscisic acid signaling in Arabidopsis . Plant Physiology 163 : 857- 866.

Wang H, Deng XW (2002) Arabidopsis FHY3 defines a key phytochrome A signaling component directly interacting with its homologous partner FAR1. EMBO Journal 21 : 1339-1349.

Wu G Z, Meyer EH, Richter A S, Schuster M, Ling Q, Schottler MA, Walther D, Zoschke R, Grimm B, Jarvis R P, Bock R (2019) Control of retrograde signalling by protein import and cytosolic folding stress. Nature Plants 5 : 525-538.

Xiong JY1, Lai CX, Qu Z, Yang XY, Qin XH, Liu GQ (2009)Recruitment of AtWHY1 839 and AtWHY3 by a distal element upstream of the kinesin gene AtKP1 to mediate 840 transcriptional repression. Plant Molecular Biology 71 :437-49.

\section{FIGURE LEGENDS}

Figure 1: Phenotypic images of wild-type (WT) barley seedlings and two independent WHIRLY1-deficient barley lines (W1-1 and W1-7) grown under standard (16h light/8h dark) photoperiod (A, B) conditions for 7 days $(\mathrm{A})$ and 14 days (B), or grown in the dark for 4 days prior to illumination $(\mathrm{C}, \mathrm{D}, \mathrm{E}, \mathrm{F}, \mathrm{G}, \mathrm{H})$. The dark pre-treated seedlings were transferred to light at the beginning of long photoperiod for $1 \mathrm{~h}(\mathrm{C}), 3 \mathrm{~h}(\mathrm{D})$, $8 \mathrm{~h}(\mathrm{E}), 16 \mathrm{~h}(\mathrm{~F}), 32 \mathrm{~h}(\mathrm{G})$ and $56 \mathrm{~h}(\mathrm{H})$. Scale bar is $1 \mathrm{~cm}$.

Figure 2: Chlorophyll a fluorescence in vivo imaging of the dark-adapted $\mathrm{Fv} / \mathrm{Fm}$ ratios of wild type and W1-7 seedlings. Seedlings were imaged during development under standard photoperiod (16h light/8h dark) conditions from the first day of leaf emergence (4 days old) up to 11 days after germination. The colour scale represents $\mathrm{Fv} / \mathrm{Fm}$ values between $0.6-0.85$. The arrows indicate the first leaf at the point of emergence and the point where achieved the highest Fv/Fm value. 
Figure 3: A comparisons of leaf pigment contents in the base, middle (mid) and tip sections of the first leaves of wild type (WT) and W1-1 and W1-7 seedlings at 7 (A) and 14 (B) days after germination and during de-etiolation following transfer to light at the beginning of the photoperiod (C). Data are presented as means $\pm \mathrm{SE}(\mathrm{n}=6)$. Different letters above the bars indicate statistically significant differences $(P<$ 0.05) analysed by two-way ANOVA with Tukey's post-hoc test.

Figure 4: A comparison of the chlorophyll a/b ratios in the base, middle (mid) and tip sections of the first leaves of wild type (WT) and W1-1 and W1-7 seedlings at 7 (A) and 14 (B) days after germination and during de-etiolation $8 \mathrm{~h}, 16 \mathrm{~h}, 32 \mathrm{~h}, 40 \mathrm{~h}$ and $56 \mathrm{~h}$ under standard photoperiod conditions (C). Data are presented as means $\pm \mathrm{SE}(\mathrm{n}=3)$. Different letters above the bars indicate statistically significant differences $(P<0.05)$ analysed by two-way ANOVA with Tukey's post-hoc test.

Figure 5: Western blot analysis of selected chloroplast proteins in the base, middle (mid) and tip sections of the first leaves of wild type (WT) and W1-1 and W1-7 seedlings at 7 (A) and 14 (B) days after germination. Proteins detected are LHCB1 and LHCB2, the small subunit of ribulose-1, 5-bisphosphate carboxylase (RBCS), the chloroplast ribosomal protein S1 (RPS1), WHIRLY1 (WHY1), the large subunit of RBC (RBCL) and the photosystem II D1 protein.

Figure 6: Western blot analysis of selected chloroplast proteins in the hours after 4 day old etiolated seedlings were exposed to light $(1 \mathrm{~h}, 3 \mathrm{~h}, 8 \mathrm{~h}, 16 \mathrm{~h}, 32 \mathrm{~h}$ and $56 \mathrm{~h})$ under standard photoperiod conditions. Proteins detected are LHCA1 and LHCB1, the chloroplast ribosomal protein RPL20 (RPL20), the ironsulphur centre of photosystem I subunit PSAC, WHIRLY1 (WHY1), and the photosystem II D1 protein.

Figure 7: The abundance of WHY1 and chloroplast-encoded transcripts measured by qPCR in etiolated seedlings following exposure to light at the beginning of the photoperiod of the $5^{\text {th }}$ day under standard photoperiod conditions (16h light/8h dark). Samples were taken following exposure to standard photoperiod conditions was for $1 \mathrm{~h}, 3 \mathrm{~h}, 8 \mathrm{~h}, 16 \mathrm{~h}, 32 \mathrm{~h}$ and $56 \mathrm{~h}$. Values are represented as means $\pm \mathrm{SE}(\mathrm{n}=3)$. Different letters represent statistically significant differences analysed by two-way ANOVA and Tukey's HSD, a posthoc test $(\mathrm{P}<0.05)$.

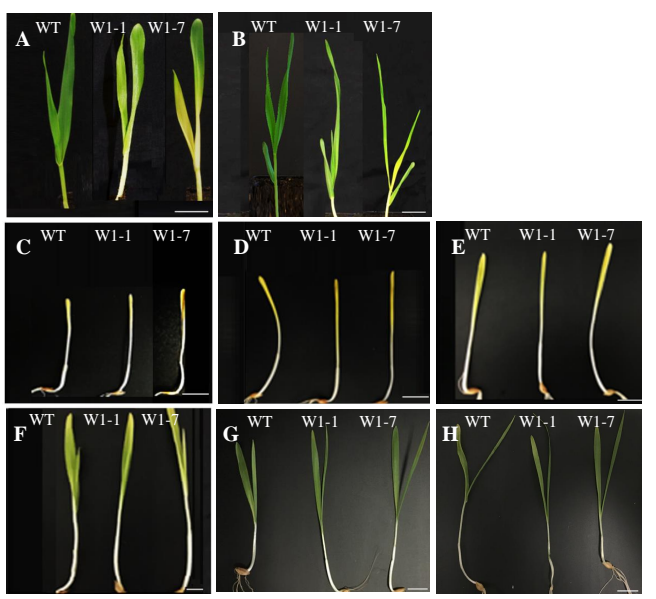



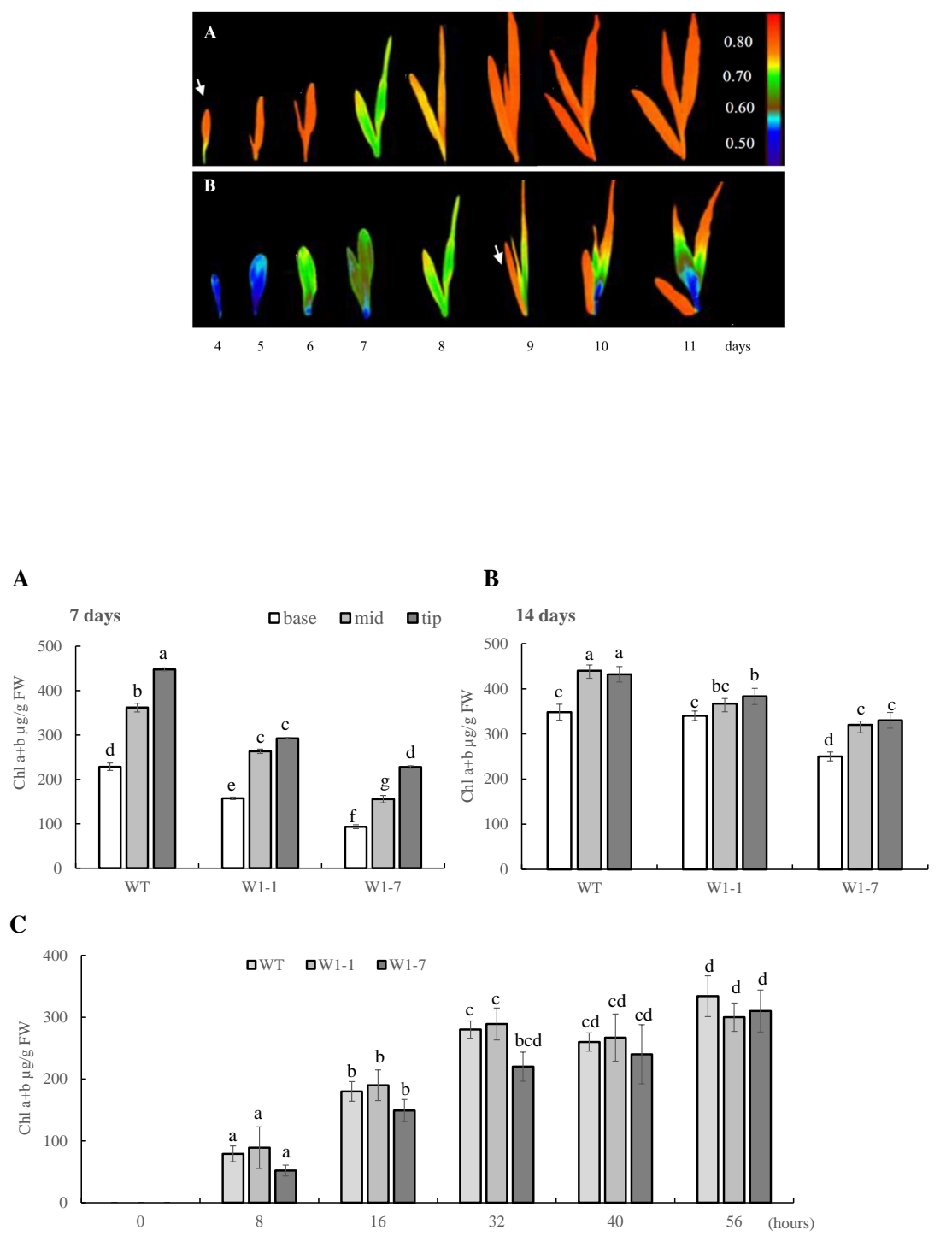

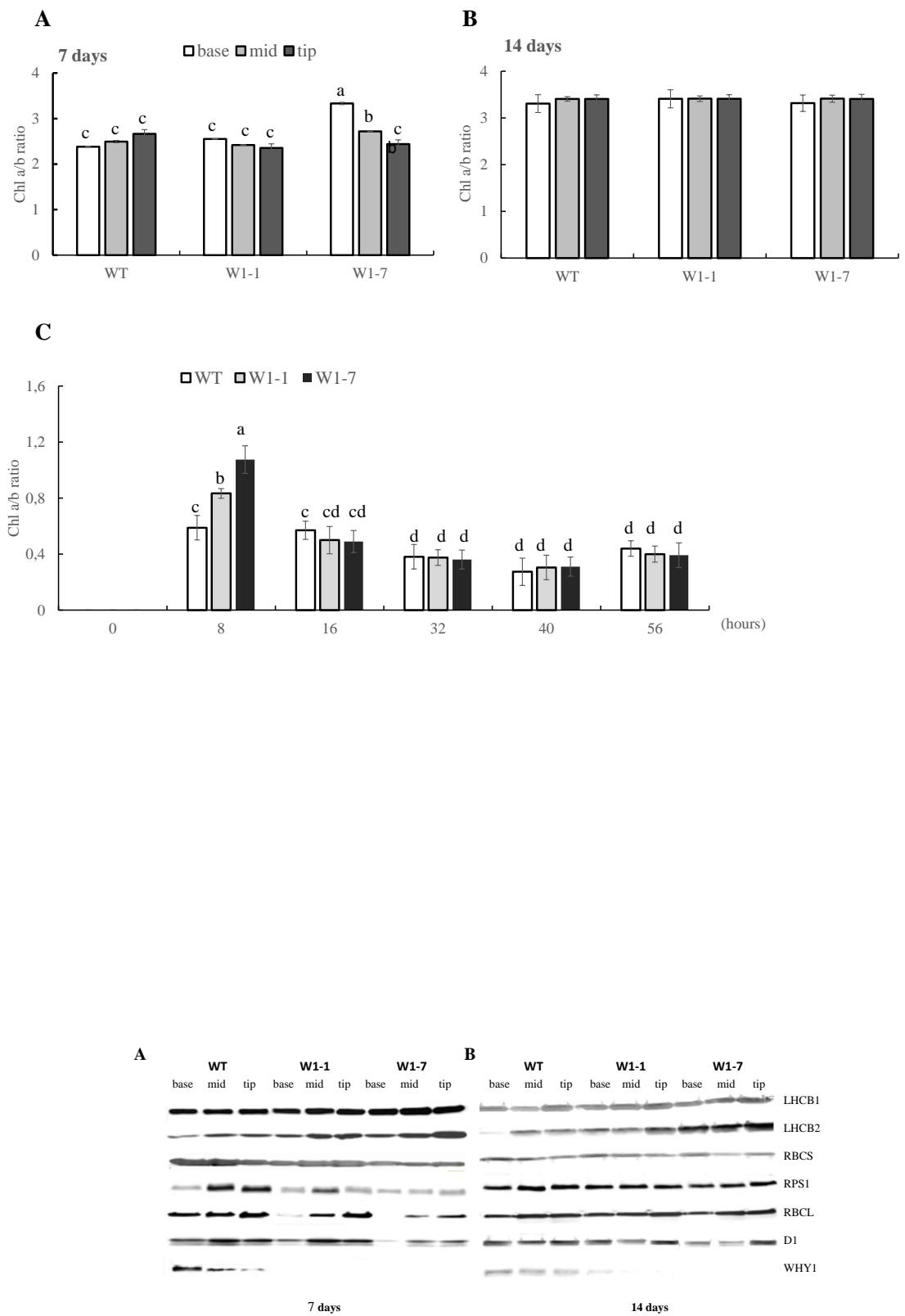

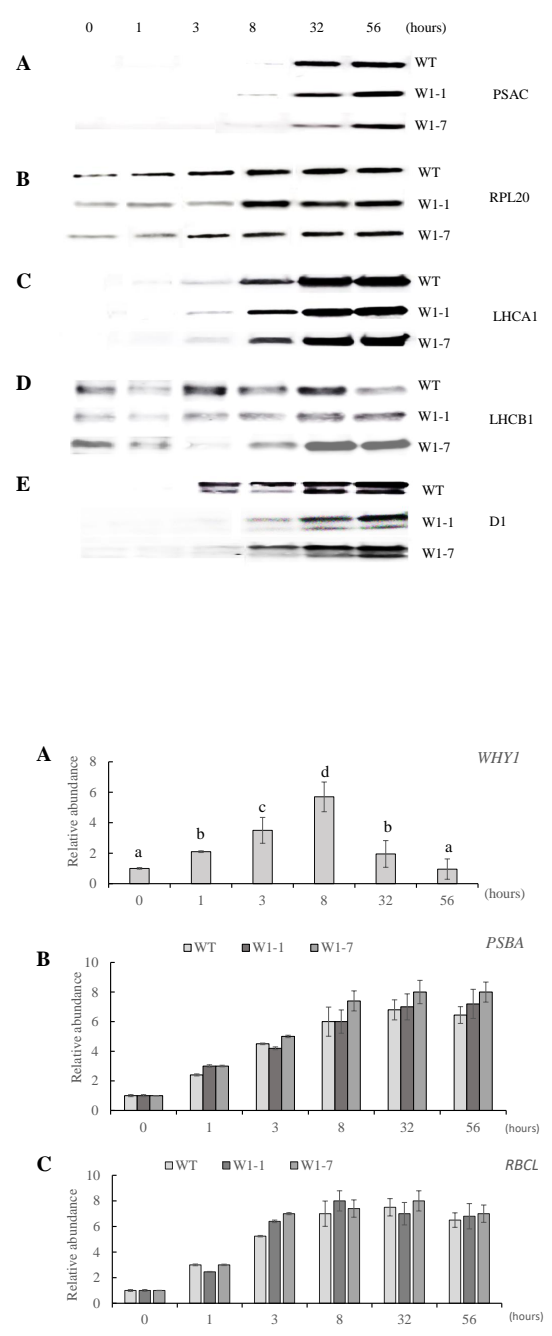